\title{
Structure Optimization of Ultra-precision Machine Tool Guide based on Isight
}

\author{
Xinkai Mao', Qingsong Hua ${ }^{1, a,{ }^{*}, \text { Lianjun Cheng }}{ }^{1}$, Qinghai Zhao ${ }^{2}$, He Xuan ${ }^{1}$ \\ ${ }^{1}$ Mechanical and Electrical Engineering, Qingdao University, Qing Dao, China \\ ${ }^{2}$ Power Integration and Energy Storage Systems Engineering Technology Center, Qingdao \\ University, Qing Dao, China \\ a Corresponding author e-mail: 89885962qq.com
}

Keywords: structure optimization; machine tool; Isight; guide rail; latin hypercube.

\begin{abstract}
The influence of the guide rail on the machining precision of the machine is great, and the optimization of the rail structure can improve the machining performance of the machine tool. Based on ANSYS Workbench simulation analysis, this study uses Isight to optimize the structure. The key dimensions of the rail structure are used to model the parameters and the optimal Latin hypercube test. The maximum deformation of the optimized rail is increased by $4.74 \%$, but the quality is reduced by $3.91 \%$, which satisfies the accuracy requirement. The optimization results verify the feasibility of the proposed method.
\end{abstract}

\section{Introduction}

The development of modern industry cannot be separated from the support of the machine, due to high precision, high speed, high stiffness and other excellent performance, CNC machine tools, especially ultra-precision CNC machine tools acquire the world's attention. GIM and others[1] use the first-order natural frequency as the goal ,induce the topology optimization for the column, and improved the dynamic stiffness of the column .Dai Lei and others[2] used POSHAPE to optimize the parameters of the rail structure and improve the rigidity and strength of the guide. As the linear guide with a compact structure, responsive, easy installation and other advantages of good performance in the machine has a wide range of applications. At the same time, as a part of the machine tool, the linear motion of the linear guide is characterized by sliding characteristics, non-linearity of stress contact, nonlinearity of contact state and elasticity. In this study, the structural strength and rigidity of the guide rail movement system are analyzed, And the use of Latin ultra-vertical method to optimize the design of the body to improve the rail shock resistance and to achieve lightweight rail.

\section{The Design of Linear Guide}

The main components of the machine includes bed, table, spindle box, column and other components including the two functions of the guide are the guiding and carrying role. Oriented high precision, long lasting accuracy are the two requirements of the machine tool guide, and also important guarantees for long-term work. Since the processing of the machine tool is affected by the vibration of the guide rail and the deformation in the axial direction (longitudinal or Y direction) of the guide rail, the two factors should be taken into account when the linear guide-slider mechanism is simulated and analyzed to optimize the quality. The specific process of simulation is that: first, SolidWorks was used to establish a three-dimensional model, and then send the model into the ANSYS, fixed rail model at both ends, apply 4000N normal stress in the middle of the guide. And the deformation of the first six modes and the amount of deformation perpendicular to the axial direction (longitudinal or $\mathrm{Y}$ direction) of the guide rail are analyzed. The position of the rail-slider mechanism on the machine is shown by the red arrow in Figure 1 below. 


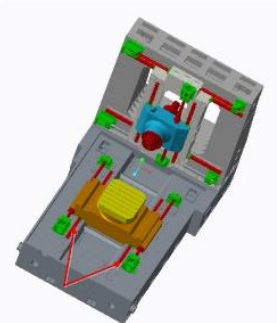

Figure 1. CNC machine tool assembly drawing.

\subsection{Guidance of the Guide Rail - Slider Mechanism}

The sizes of the rail-slider mechanism have been designed to meet the test requirements. Now the rail sizes are optimizing. Guide selects bearing steel, slider selects alloy steel, the material parameters shown in Table 1 below ${ }^{[3]}$.

Table 1. Material parameter table.

\begin{tabular}{cccc}
\hline Material & Elasticity Modulus(GP) & Poisson Ratio & Density $(\mathrm{kg} / \mathrm{m} 3)$ \\
\hline Bearing Steel & 208 & 0.3 & 7900 \\
Alloy Steel & 206 & 0.28 & 7800 \\
\hline
\end{tabular}

Guide rail size parameters are: length L: 810; width w: 50; thickness d: 45

It is known that the Z-axis direction of the machine is double bearing, and the maximum allowable load of the worktable is $8000 \mathrm{~N}$. Therefore, the maximum load of single-side guide is $4000 \mathrm{~N}$ and the maximum deformation is 1.5 ( $\mathrm{mm}$, the following dimensions are not specified). The working frequency of this machine is about $136 \mathrm{~Hz}$. In the ANSYS simulation results, the first three frequencies $\mathrm{P} 1, \mathrm{P} 2$ and $\mathrm{P} 3$ are $89.088 \mathrm{~Hz}, 98.303 \mathrm{~Hz}$ and $244.03 \mathrm{~Hz}$ respectively. In order to avoid the resonance of the machine tool, the three frequencies are selected as the constraint condition to avoid the frequency in the $\mathrm{Pw}(90 \%-110 \%)$ range

\subsection{Establish an Optimized Mathematical Model}

1. Optimal design parameters

Width w, initial value: 50, adjustment range: 48-50; thickness d, initial value: 45 , adjustment range: 40-45

2. Restrictions

$\mathrm{Y}$ deformation amount $\mathrm{P} 4$, the maximum deformation is 1.5 ;

First order frequency P1, less than or equal to $122.4 \mathrm{~Hz}$;

Second order frequency $\mathrm{P} 2$, less than or equal to $122.4 \mathrm{~Hz}$;

Third order frequency P3, greater than or equal to $149.6 \mathrm{~Hz}$;

3. Objective function

The objective function of the model is that the guide rail minimizes the mass $\mathrm{M}$ under the above constraint conditions.

4. Mathematical model

Min F(M)

$$
\begin{gathered}
48<\mathrm{W}<50 \\
40<\mathrm{d}<45 \\
0<\mathrm{P} 1<122.4 \\
0<\mathrm{P} 2<122.4 \\
149.6<\mathrm{P} 3<245
\end{gathered}
$$

\section{Isight Collaborative Optimization Design}

\subsection{Experimental Design Method}

In order to ensure the accuracy of the test and to minimize the calculation time, the optimal Latin hypercube design (Opt LHD) method is adopted in this study. At the end of the 1970s, random Latin hypercube design (LHD) began to apply. The calculation principle is as follows: In the n-dimensional space, each coordinate space is divided into $\mathrm{m}$ intervals, and randomly selected $\mathrm{m}$ points, to ensure 
that each factor of a factor is studied once. That is, $\mathrm{n}$-dimensional space $\mathrm{m}$ sample points of the Latin hypercube design, that is, $m * n$ LHD [4].

Latin hypercube design has the following advantages

(1) Effective space filling ability. As shown in Figure 2 and Figure 3, the LHD needs fewer test points in the same design space than the full factorial design (FFD).

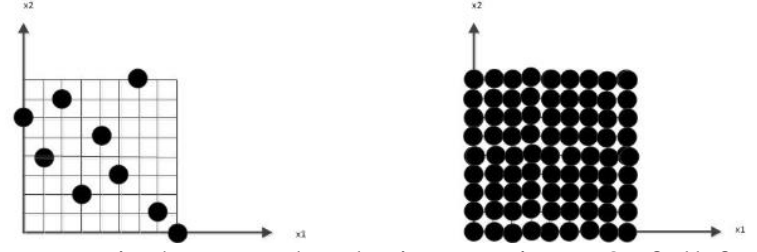

Figure2. random Latin hypercube design. Figure3. full factorial design.

(2) High precision fitting is better for nonlinear response. As the number of test methods can be artificially controlled, it is suitable for dealing with the optimization problem of large amount of data. As shown in Fig. 2 and Fig. 4, the Latin hypercube design can fit a higher order nonlinear relationship with the same number of points as compared to Orthogonal Arrays (OA).
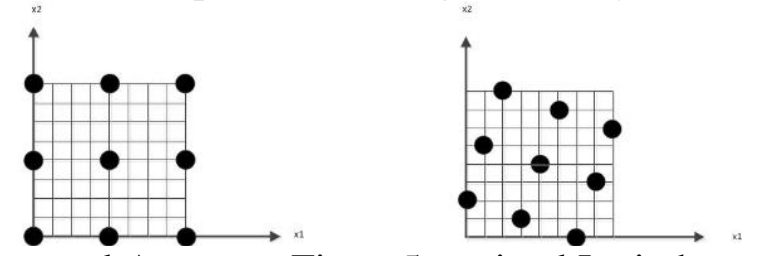

Figure4. Orthogonal Arrays. Figure5. optimal Latin hypercube design.

Latin hypercube design since the promotion in the major research areas have been widely used, but its own shortcomings. The randomness of the sample selection of the LHD determines the nonrepeatability of the test, so the test results are difficult to reproduce. At the same time, as the number of test levels increases, the possibility of loss of some areas of the design space increases gradually, and the accuracy and fitting ability of the test decreases ${ }^{[5]}$. Based on this, the Opt LHD method was chosen. As shown in Fig. 2 and Fig. 5, the modified Latin hypercube design can make all the test points evenly distributed evenly in the design space, with good balance and spatial filling ${ }^{[6]}$.

\subsection{Integration Process}

ANSYS Workbench is an internationally renowned finite element analysis software that can deal with complex problems such as heat, electricity, fluid and other physical field coupling ${ }^{[7]}$. Isight is a collaborative simulation software, you can directly call the common software in various fields. Figure 6 shows the optimal flow chart for Isight's use of professional components to integrate the Workbench. Isight can help users greatly shorten the development cycle, improve product quality, reduce product costs, and it also has an outstanding performance in the process of integration and optimization of the design field ${ }^{[8]}$.

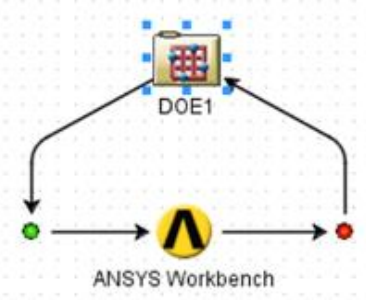

Figure6. Isight optimization process.

Isight in the call Workbench implementation of the simulation process, due to the optimization of the parameters of the selected settings, optimization process will appear part of the test point does not meet the design requirements of the situation, this test point is dead pixels. The software will not process the set of parameters, but will still call the last generated result file so that the optimization continues until the optimal solution satisfies the constraint. 


\section{Optimization Results}

According to the optimization scheme, 60 sampling points are selected to carry out the optimal Latin hypercube test, as shown in Figure 7, the volume iterative optimization diagram and the maximum vertical (Y) deformation in Figure 8, the optimal solution is shown in step 53 Green point), and the minimum volume of the rail appears at step 60, which is smaller than the optimal solution given by the software, but the point exceeds the Y-direction maximum deformation constraint requirement, that is, the point does not meet the stiffness requirement of the structure, give up.

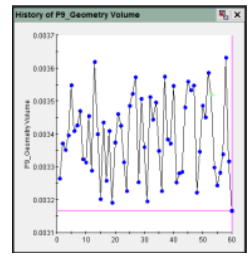

Figure7. the volume iterative optimization diagram.

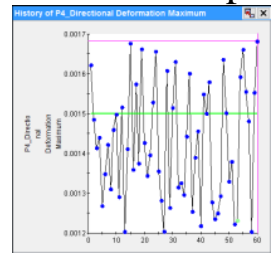

Figure8. the maximum vertical (Y) deformation.

Figure 9 shows the two-dimensional constraint graph of volume for w, d, and Figure 10 is a twodimensional constraint graph of Y-direction maximum deformation amount with respect to $\mathrm{w}, \mathrm{d}$. The distribution of the 60 sampling points in the optimal Latin hypercube test is shown in Fig. 9 and Fig. 10 , and due to the constraints of the first, second, third order frequency and the maximum vertical deformation constraints, the optimal solution point occurs in the upper left corner of the circle.

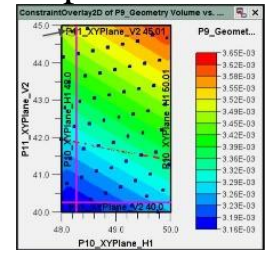

Figure 9. the two-dimensional constraint graph of volume for $\mathrm{w}, \mathrm{d}$.

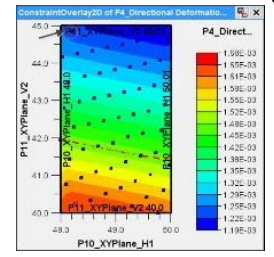

Figure 10. the two-dimensional constraint graph of Y-direction maximum deformation amount with respect to $\mathrm{w}, \mathrm{d}$..

After optimization, the parameters of the guide rails change, optimize the parameters before and after the comparison shown in Table 2 below. The volume decreased to $0.0033136 \mathrm{~m} 3$, compared to $0.0036635 \mathrm{~m} 3$ before optimization, the quality decreased $3.91 \%$; $Y$ to the maximum deformation from $0.0011752 \mathrm{~m}$ rose to $0.0012309 \mathrm{~m}$, up $4.74 \%$, within the allowable range of deformation, satisfied the stiffness, strength design requirements.

Table2. Comparison of parameters before and after optimization.

\begin{tabular}{cccccccc}
\hline parameters & $\mathrm{W}$ & $\mathrm{d}$ & $\mathrm{P} 4$ & $\mathrm{P} 1$ & $\mathrm{P} 2$ & $\mathrm{P} 3$ & $\mathrm{~V}$ \\
\hline before optimization & 50 & 45 & 0.0011752 & 89.088 & 98.303 & 244.03 & 0.0036635 \\
after optimization & 48.204 & 44.84 & 0.0012309 & 88.735 & 94.778 & 243.05 & 0.0035201 \\
\hline
\end{tabular}

\section{Conclusion}

1) The finite element analysis software of Ansys Workbench is used to check the stiffness and strength of the ultraprecision CNC machine tool. On this basis, the result of finite element 
analysis is used to optimize the guide rail structure by Isight collaborative optimization software. The maximum deformation of the optimized rails increased by $4.74 \%$, but within the allowable range, the quality was reduced by $3.91 \%$ and the production cost was reduced.

2) The finite element analysis and collaborative optimization method used in this study have great versatility and practical value for the checking and optimization of mechanical parts.

\section{Acknowledgments}

This work was financially supported by National Science and Technology major projects funded projects (2015ZX04005001).

\section{References}

[1]. GIM T, LEE C H, HA J Y. Machine Tool Technologies for High Performance Machines[C]// International Conference on Smart Manufacturing Application, Korea, 2008: 230-233.

[2]. Lei Dai, Zhenqun Guan, Julin Shan, et al. Three - Dimensional Parametric Shape Optimization Design of Machine Tool Structure [J]. Journal of Mechanical Engineering, 2008, 44(5): 152-159.

[3]. Mechanical Engineering Materials Data Handbook Editorial Board. Mechanical Engineering Material Performance Data Sheet [M]. Beijing: Machinery Industry Press, 1994: 42-43.

[4]. Yuyang Lai. Isight parameter optimization theory and examples [M]. Beijing: Beijing University of Aeronautics and Astronautics Press, 2012:118-119.

[5]. Ming Cong, Too Hag, Qiang Zhao. Fuzzy Multi-Objective Optimization of Sliding Rack Based on Six Sigma and Goal Driven[c]2010 International Conference on Mechanic Automation and Control Engineering, Wuhan. China. 2010(6): 556-559.

[6]. Zhengwen Jiang, Shui Wan, Minghong Li, et al. Hybrid Simulation Method and Its Application in Structural Reliability Analysis[J].Journal of Zhejiang University(Engineering Edition), 2015, (4):782-791.

[7]. Jianfang Xia, Nanhai Ye, The Principle of Finite Element Method and the Application of ANSYS[M]. National Defense Industry Press, 2011.10.

[8]. Jianzhao Lu, Guofu Yin, Ling Wang. Research on the CAD/CAE integrated optimization method of machine tool structures based on Isight [J]. Modular Machine Tool \& Automatic Manufacturing Technique, 2013(2): 22-24(in Chinese). 